\title{
Mesures géophysiques pour l'analyse des glissements de terrain
}

M. FRAPPA

Centre de développement des qéosciences appliquées

Université Bordeaux I

33405 Talence

thomaslebourg@hotmail.com. frappa@cdga.u-bordeaux.fr

T. LEBOURG

Université de Nice Sophia-Antipolis Laboratoire Géosciences Azur

UMR 6526

250, rue Albert-Einstein 06560 Valbonne lebourg@geoazur.unice.fr
Cet article présente différentes méthodes de prospection géophysiques utilisées pour la caractérisation, la reconnaissance de paramètres et les facteurs liés aux glíssements de terrain. Nous nous sommes principalement intéressés à la recherche du plan de rupture limitant la base du glissement, du système topographique complexe caché (ou cryptorelief) et aux systèmes de chenalisation interne. Les méthodes de prospection utilisées sont le panneau électrique (pseudosections), la polarisation spontanée (PS), le profilage électromagnétique (Slingram, EM31) et le sondage électromagnétique en domaine temporel (TDEM). La prospection géophysique montre qu'elle permet de renseigner sur les limites géométriques où les glissements de terrain peuvent survenir et elle permet également une quantification des paramètres géométriques et hydrauliques utilisés dans les modèles numériques de stabilité.

Mots-clés : instabilité, glissements de terrain, prospection électrique, prospection électromagnétique, chenalisation. surface de rupture, cryptorelief.

\section{Geophysical survey for landslides analysis}

The purpose of this paper is to present different results of geophysical prospecting to investigate factors of instability characterising landslides. We are interested here with the slicing plan, the complex topographic systems and the interconnected channel system. The geophysical prospecting methods used here are the electrical survey (pseudo-sections), the spontaneous polarisation (SP), the electromagnetic survey (Slingram, EM31) and the time domain electromagnetic survey (TDEM). The geophysical prospecting dives a knowledge on the boundary of areas where natural hazards can occur, and allows also to quantify input parameters as the geometry of failed area or hydraulic parameters used for numerical models of stability.

Key words : instability, landslides, electrical survey, electromagnetical survey, channel, sliding surface, cryptorelief. 


\section{Introduction}

Cet article présente de nombreux résultats géophysiques d'un projet, lancé depuis quatre ans, sur la reconnaissance des instabilités de versants montagneux dans les Pyrénées-Atlantiques. C'est plus particulièrement la reconnaissance de caractéristiques relatives aux instabilités que nous proposons de développer dans cet article. Dans un premier temps, on traitera de la définition des caractéristiques associées aux facteurs d'instabilités et identifiables par mesures indirectes, puis dans un second temps, on présentera les différentes mesures géophysiques réalisées, ainsi que les résultats obtenus au reqard des indices de terrain. Les facteurs favorables à l'initialisation des glissements de terrain, retenus dans ce travail, sont : les surfaces de rupture, la topographie sous-jacente complexe ainsi que la chenalisation interne favorisant la circulation de l'eau et les variations des pressions interstitielles. Au travers de ces expérimentations et applications, la prospection géophysique, au sens large, s'avère un excellent moyen d'investigation dans des secteurs à géologie complexe et difficile d'accès, et elle permet d'obtenir des renseignements sur de nombreux facteurs conditionnant les glissements de terrain.

\section{Localisation géographique}

Le secteur d'étude est géographiquement situé dans les Pyrénées-Atlantiques, la vallée d'Aspe (Fig. 1). C'est une zone de moyenne et haute montagne dont les sommets culminent à $2200-2300 \mathrm{~m}$ d'altitude et les vallées s'étagent entre 400 et $1000 \mathrm{~m}$ d'altitude.

La région étudiée se situe, pour l'essentiel du travail, dans la haute vallée d'Aspe, au Sud de Pau, entre le Fort du Portalet et le col du Somport (frontière avec I'Espagne) (Fig.1). En haute vallée d'Aspe, le terrain est limité, au sud, par les falaises calcaires et, au nord, par les reliefs calcaires du Dévonien au Fort du Portalet. immédiatement au sud du village d'Etsaut (Fig. 2).

Ce secteur d'étude fait environ $40 \mathrm{~km}^{2}$ (5 km de large et $8 \mathrm{~km}$ de long). Il limite géographiquement la ligne de partage des eaux du gave d'Aspe (cóté français) avec du côté espagnol, le rio Aragon.

Les cours d'eaux, orientés E-W, suivent les principales directions structurales correspondant aux grandes structures plicatives du substratum régional.

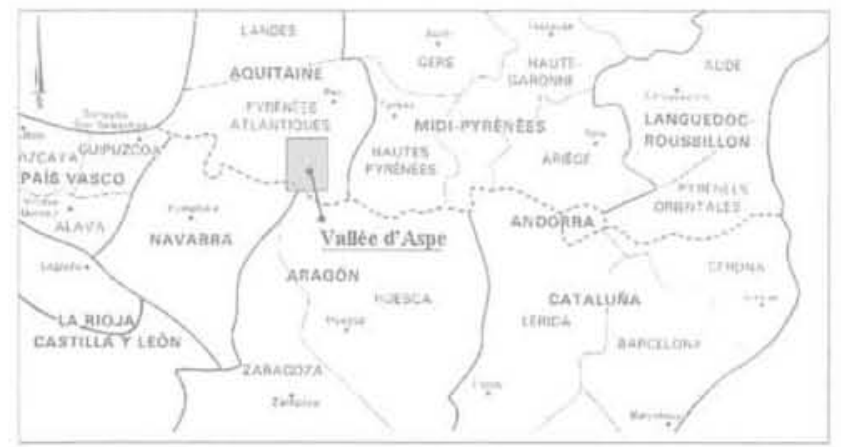

FG.1 Localisation géographique de la vallée d'Aspe.

Map of the studied area.

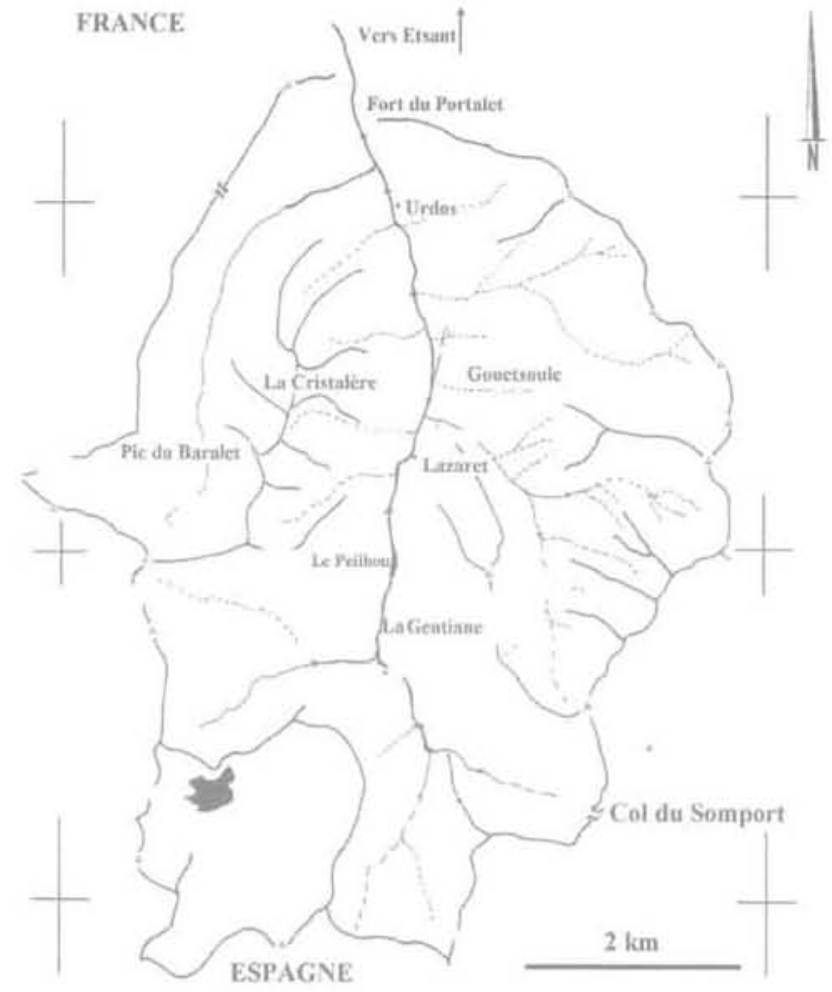

FIG. 9 d'Aspe. Aspe valley toponymical map.

\section{3}

\section{Caractéristiques identifiables par mesures indirectes}

Parmi les nombreux éléments de reconnaissances qui interviennent dans la compréhension des instabilités de terrain, on s'est intéressé aux facteurs difficilement observables et quantifiables. Parmi ces facteurs et caractéristiques qui nécessitent une identification par la mesure indirecte, on peut définir :

- le cryptorelief (relief du substratum caché par les formations superficielles) mis en évidence comme étant étroitement lié aux surfaces de rupture (Lebourg et al., 1999, 2000; Flageolet et al., 1999);

- les surfaces de glissement et le contraste de propriétés physiques entre une masse glissée et le massif en place (Bogoslovsky et Ogilvy, 1977; Caris et Van Asch, 1991 ; Lebourg et al., 1999, 2000 ; Gallipoli et al., 2000); - la circulation interne de l'eau (Durville et al., 1992; Lebourg et al., 1999, 2000; Fabre et al., 2000; Wong, 2000).

\section{1}

\section{Cryptorelief ou le relief du substratum caché}

L'étude des différentes formes de reliefs engendrées par l'action érosive des glaciers (Menzies, 1996) montre le rôle majeur que peut jouer la géomorphologie du substratum dans l'apparition de surfaces de rupture préférentielles. Il s'agit d'une géomorphologie bien 
particulière pour qualifier les formes cachées d'érosion sous des formations meubles. Nous l'appelons : cryptorelief.

La présence de structures concaves " en gouttière \# affectant le substratum, sur lequel reposent les formations morainiques susceptibles de glisser, est un facteur qui aggrave les risques de glissement. Pour illustrer ce fait, comparons deux cas de glissements de terrain, l'un avec un substratum tabulaire recouvert par de la moraine et l'autre avec un substratum de forme concave.

Dans les deux cas de figure la forme concave du glissement de terrain n'aura pas la même géométrie (Fig. 3) et les matériaux sollicités dans la résistance au cisaillement seront différents. Il est intéressant de remarquer que dans le premier cas de figure $(\mathrm{Cx}$ 1 à Cx_3) la résistance au cisaillement se fait essentiellement au contact substratum/moraine, alors que dans le second cas de figure (Mo_1 à Mo_3) la résistance au cisaillement est celle de la moraine (paramètres mécaniques de la moraine). D'autre part, en supposant que la surface de rupture affecte intégralement le matériel morainique, nous avons constaté que le changement de la géométrie de la surface de rupture, engendrait une variation significative du coefficient de sécurité (Lebourg, 2000), En utilisant un code de calcul de stabilité par la méthode de Spencer étendue à un modèle $3 \mathrm{D}$, on a pu mettre en évidence que la prise en compte du cryptorelief diminuait la valeur du coefficient de sécurité global (Lebourg, 2000). Nous passons ainsi, pour le profil de calcul de stabilité, d'un coefficient $F=1,01$ sans prise en compte de la géométrie due au cryptorelief, à $\mathrm{F}=0,93$ pour le modèle intégrant le cryptorelief. Cet exemple montre l'importance de la géomorphologie en « gouttière » qui est un facteur à intégrer dans le calcul de l'instabilité. Il convient aussi d'évaluer l'importance de cette forme concave, l'épaisseur du remplissage et les différentes hétérogénéités de dépôt. Ces autres facteurs sont analysés soit par une méthode directe, la cartographie de terrain, soit par des méthodes indirectes : reconnaissance électrique ou électromagnétique.

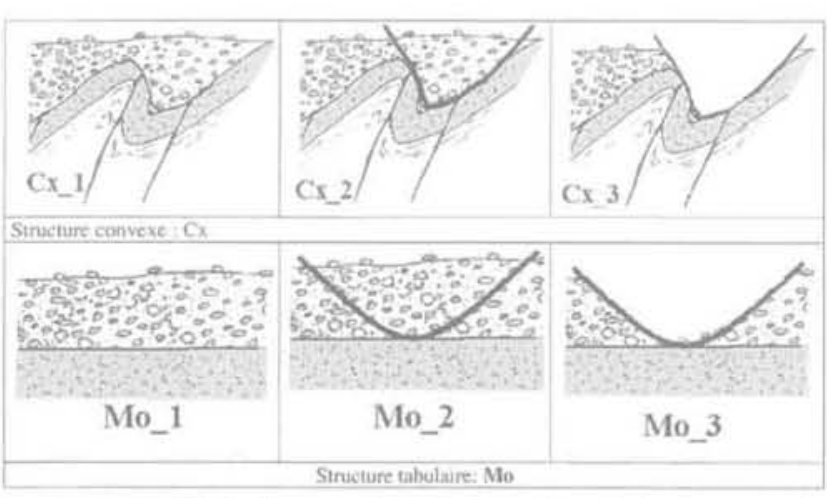

FIG. 3 Influence de la forme du substratum sur le glissement de terrain.

Substratum morphology influence on the landslide.

\section{Surface de glissement et contraste de propriétés physiques entre une masse glissée et le massif en place}

La mesure du contraste de propriétés physiques entre le massif en place et une masse instable est facilement quantifiable par les mesures de résistivité. Ce contraste est généralement généré par une discontinuité lithologique ou une modification de la structure physique (porosité, perméabilité) au niveau de la zone de glissement. Si l'on considère un glissement dans un terrain homogène, le remaniement interne de la masse en mouvement engendre un contraste de résistivité élevé entre le massif en place et la masse en mouvement. Il y a alors eu changement de la structure, suffisamment important pour augmenter la porosité de façon conséquente. Cette variation positive de la porosité induit une augmentation de la teneur en eau du sol en mouvement par rapport au massif en place. Bogoslovsky (1977), sur des glissements près des bords des rives de la Volga, a distingué la limite entre la masse allochtone (5-10 $\Omega \mathrm{m})$ et la masse autochtone (30$40 \Omega \mathrm{m}$ ) dans une même formation alluvionnaire. Caris et Van Asch (1991), ont obtenu des résultats similaires lors de l'étude d'un glissement circulaire affectant une masse homogène de Terre noires dans les Alpes. La masse instable avait une résistivité d'environ 10-50 $\Omega \mathrm{m}$ tandis que la zone stable avait une résistivité de $75-150 \Omega \mathrm{m}$.

Selon la méthode de prospection utilisée, il est possible de localiser la zone affectée par la présence d'une surface de rupture dont la localisation est plus ou moins précise (Lebourg et al., 1999; Gallipoli et al., 2000). Ainsi, des pseudo-sections de résistivité, réalisées sur des profils perpendiculaires à des glissements translationnels, ont permis de modéliser la géométrie en trois dimensions d'un glissement intra-morainique dans les Pyrénées (Lebourg et al., 1999).

\section{3}

\section{Circulation interne de l'eau}

La variation des conditions hydrauliques est l'une des principales causes de glissement de terrain et son action dans la rupture de l'équilibre se manifeste de plusieurs façons et à différents stades (Durville et al., 1992). Comme cela a été présenté précédemment, le cryptorelief, facteur d'instabilité, est associé à un héritage de l'érosion glaciaire, qui a aussi généré dans certaines formations comme les moraines, un système de chenalisation interne complexe, hérité des différentes phases de retrait glaciaire. Sur de nombreux secteurs d'étude (Lebourg et al. 1999, 2000; Fabre et al., 2000) on a remarqué que la circulation d'eau dans les moraines était guidée par un système complexe de chenalisation interne et d'alimentation à la faveur de la fracturation des substratums. Cette observation, qui a aussi été faite dans les dépôts volcaniques hétérogènes instables de la région de Tokyo (Wong, 2000), montre que l'eau circule d'une façon aléatoire et vraisemblablement guidée par l'hétérogénéité du milieu. Il semble qu'il existe dans les moraines un système d'alimentation plus complexe. C'est principalement sur la base de nombreuses observations de terrain, de forages et de sondages électriques et électromagnétiques que nous allons préciser certains chemins de circulation préférentielle de l'eau dans les formations instables. 
L'hypothèse de la chenalisation interne est le fruit de nombreuses observations de terrain, étayées par les interprétations de la prospection électrique réalisée pour la reconnaissance des surfaces de rupture. Nous avons remarqué, sur l'ensemble des versants recouverts par les dépôts morainiques, soit des sources, soit des zones localement humides affectées par des suintements. Ces zones humides sont caractérisées par une différence granulométrique entre l'encaissant et le drain. Les drains sont dépourvus de particules fines et sont constitués, pour l'ensemble, d'éléments grossiers. Une reconnaissance en profondeur des drains montre qu'ils sont limités dans l'espace et qu'ils sont, d'après une analyse comparative réalisée sur les glaciers actuels, le résultat d'un processus de granoclassement différentiel, réalisé lors des phases de retrait fluvioglaciaire (Fig. 4) ou lors de générations de laves torrentielles post-retrait glaciaire (Coussot, 1993; Sorgi-de-Gennaro, 1999).

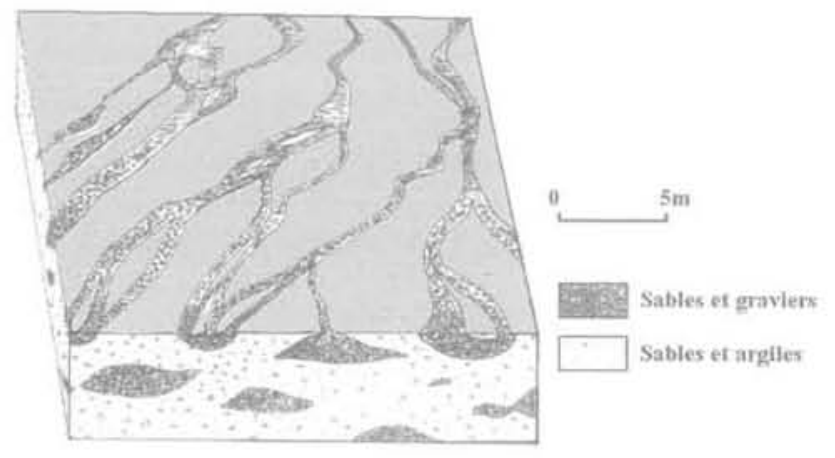

FG.4 Schéma de la chenalisation et du granoclassement différentiel lors du retrait glaciaire.

Drainage channel systems in the tills after the glacial retreat process.

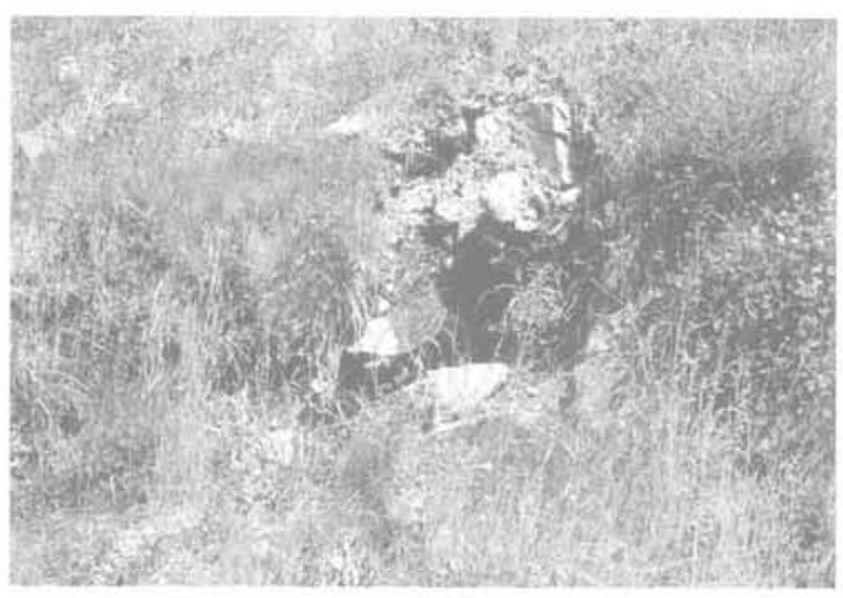

Flo. 5 Photographie d'un chenal à l'affleurement. Photography of a drainage channel on a till outcrop.

\section{4}

\section{Les différentes méthodes géophysiques et leurs résultats}

Les différentes caractéristiques qui viennent d'être analysées se traduisent par des variations de para- mètres physiques qui peuvent être appréhendées depuis la surface, par des méthodes géophysiques.

\section{1}

\section{Les mesures de résistivité : pseudo-sections}

Les pseudo-sections de résistivité constituent un outil assez bien adapté à la mise en évidence des structures du sous-sol dans ces environnements. L'inversion à l'aide de logiciels tels que RES2DINV (Lohke, 1996) restitue dans ce cas d'une part les différences de teneur en eau et d'autre part la morphologie du substratum. L'hétérogénéité des matériaux ne facilite pas la procédure d'inversion. Le glissement du Peilhou (Fig. 2) est la meilleure illustration de l'influence de la structure du substratum ou cryptorelief sur un ensemble de glissements de terrain complexes, dans le sens où nous avons affaire à une série de trois glissements emboités. Le premier panneau, constitué par les moraines, est limité en profondeur par une surface de rupture matérialisée par le contact moraine-substratum. Une campagne de prospection électrique a mis en évidence la présence d'une cuvette (cryptorelief) dont l'axe est dans le sens de la pente (Figs. 6 et 7). L'épaisseur des moraines dans cette zone varie de $10 \mathrm{~m}$ au Sud, près du tunnel du Peilhou (Fig. 2) à 30-35 m côté nord (Figs. 6 et 8), Les deux autres panneaux correspondent à un glissement de deux structures profondes, où les formations du Carbonifère ont été sollicitées par la tectonique et ont engendré des surfaces de ruptures à la faveur des différentes discontinuités lithostratigraphiques et mécaniques.

Sur les sections de résistivité (Fig. 6) nous constatons que les zones les plus conductrices correspondent à des drains ou chenaux d'alimentation en eau (vérifiés sur le terrain et dans le tunnel ferroviaire du Peilhou) et les zones les plus résistantes au substratum (remontée vers $40 \mathrm{~m}$ et $90 \mathrm{~m}$. Fig. 6). Nous pouvons ainsi observer, sur la section de résistivité et sur son interprétation, l'allure générale de la morphologie cachée du substratum (cryptorelief, Fig. 7).

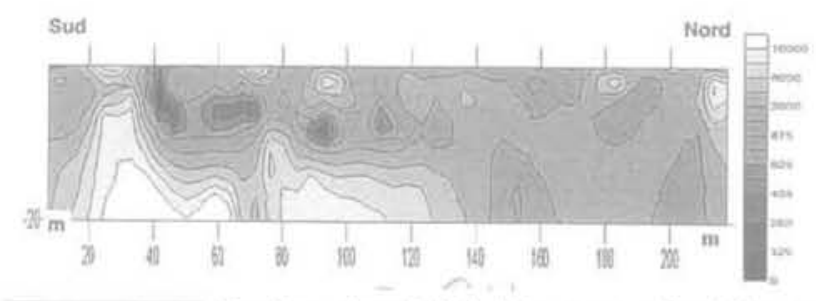

FiG.6 Section de résistivité perpendiculaire au glissement du Peilhou.

Peilhou landslide perpendicular resistivity cross section.

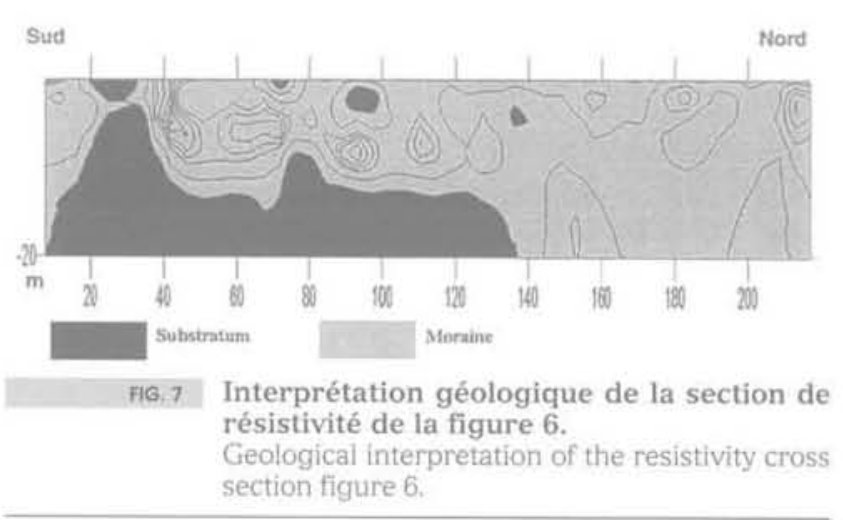




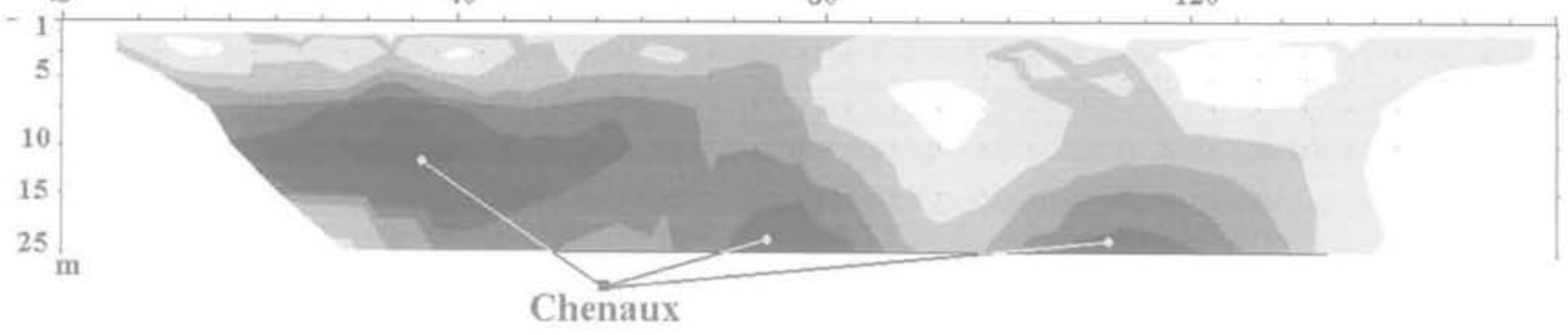

FIG 8 Section de résistivité (pôle-dipôle) du site du Peilhou et localisation des chenaux. Peilhou resistivity pseudo section (pole-dipole) and localisation of drainage channels.

Les zones correspondant aux résistivités les plus faibles ont permis de mettre en évidence la présence d'un système de chenalisation interne favorisant l'alimentation en eau de la base du glissement. Les sections de résistivité permettent d'identifier des anomalies conductrices localisées correspondant à des chenaux qui sont associés à des arrivées d'eau massives observées dans le tunnel du Peilhou. La section de résistivité pôle-dipôle (Figs. 6 et 8) montre trois zones de fortes conductivités assimilées à une circulation d'eau (Bogoslovsky et Ogilvy, 1977; Lebourg et al., 1999, 2000; Gallipoli et al., 2000).

En effet, les observations de terrain nous ont permis de constater qu'il existe un système complexe d'alimentation en eau régi par la fracturation du substratum ainsi que par des chenaux interconnectés.

Ce sont les différentes phases de retrait glaciaire qui ont créé un système de chenalisation, avec granoclassement et départ des particules les plus fines donnant ainsi des axes de drainages préférentiels. Ces derniers peuvent provoquer une mise en charge importante propice à l'instabilité, occasionnant une rupture sous la forme de glissements de terrain et/ou de coulées. Dans d'autres conditions, glissement de Gouetsoule (Fig. 2) des sections de résistivité transversales mettent en évi- dence la surface de glissement, caractérisée par une forte teneur en eau responsable des faibles résistivités rencontrées.

\section{2}

\section{La polarisation spontanée : PS}

Au cours de la réalisation de l'une des pseudo-sections implantées en travers du glissement de Gouetsoule (Fig. 2), la polarisation spontanée (PS) a été relevée pour les différents niveaux constituant la pseudo-section (Fig. 9). Ces résultats sont présentés sous formes de 5 séries de mesures à la figure 10. L'élévation progressive de la PS correspond au fait que, de la série 1 à la série 5, l'écart entre électrodes de mesure augmente chaque fois de $5 \mathrm{~m}$. Ces mesures confirment l'existence d'une limite à $140-145 \mathrm{~m}$ de l'origine. La limite ainsi déterminée coïncide exactement avec celle qui peut être déduite de la pseudo-section. La chute brutale de la PS au passage de cette limite caractérise une infiltration d'eau (Ogilvy et al., 1968, 1970, 1979; Bogoslovsky et al., 1973). En réalité, sur le terrain nous notons localement la présence d'une zone humide entretenue par une intense circulation d'eau.

Model resistivity with topography

iteration 4 RMS error $=23 \mathrm{E}$

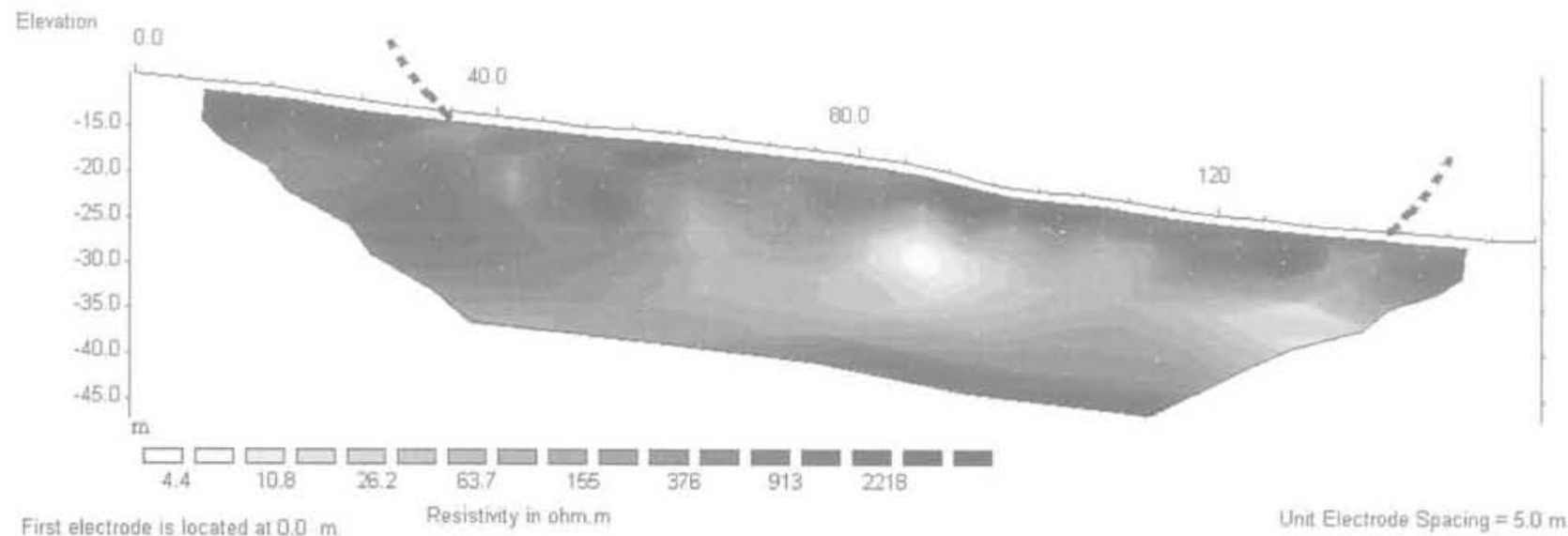

Last electrode is located at $1550 \mathrm{~m}$

FG.9 Section de résistivité (dipôle-dipôle) du site de Gouetsoule.

Gouetsoule resistivity pseudo section (dipole-dipole). 
Les cing séries de mesures enregistrées (Fig. 10) présentent une très bonne cohérence, même dans les détails les plus fins. La PS, qui n'est pas enregistrée lors de la réalisation des pseudo-sections, constitue un supplément d'information intéressant qui mériterait d'être expliquée jusque dans les plus petits détails.

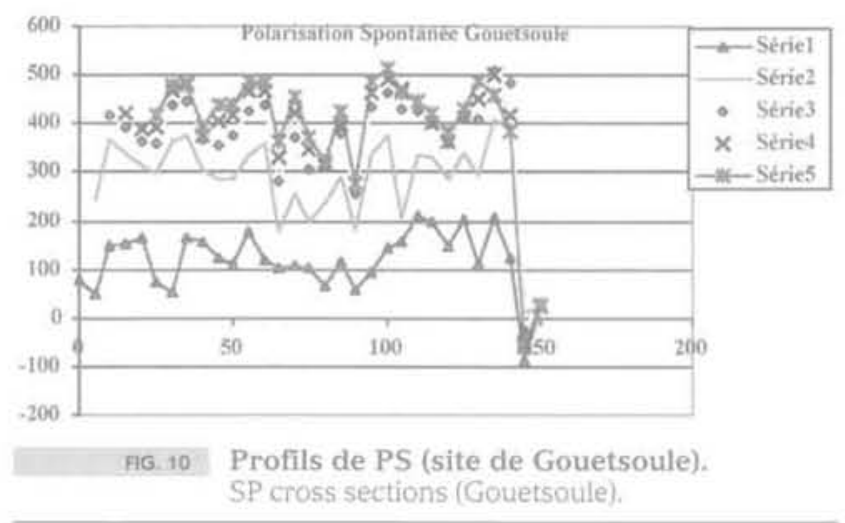

\section{3}

\section{Profilage électromagnétique (slingram)}

Le profilage électromagnétique en configuration ("slingram » à faible valeur du nombre d'induction (Mac Neil, 1980) a fait ses preuves, dans de nombreux domaines, pour l'étude de la subsurface. Nous avons donc tenté de l'utiliser à la faveur de l'ouverture d'une nouvelle piste traversant le glissement de Gouetsoule. Les mesures ont été effectuées en champ vertical et en champ horizontal, pour lesquelles les profondeurs d'investigation sont respectivement de l'ordre de 6 et $3 \mathrm{~m}$. Les différences de conductivité électrique à ces différentes profondeurs permettent de distinguer la partie stable de celle qui est en mouvement.

En dehors des anomalies dues à des aménagements divers : pylône électrique $(125 \mathrm{~m})$, buse $(210 \mathrm{~m}$ de longueur), la conductivité est identique pour les deux modes de mesure dans l'emprise du glissement, soit environ $8 \mathrm{mS} / \mathrm{m}$ (Fig. 11). En revanche, en dehors, audelà de $160 \mathrm{~m}$, la conductivité en champ vertical est toujours supérieure à celle mesurée en champ horizontal, ce qui traduit une plus grande proximité du substratum schisteux. L'épaisseur plus importante des dépôts morainiques affectés par le glissement pourrait être à l'origine des différences observées par cette méthode.

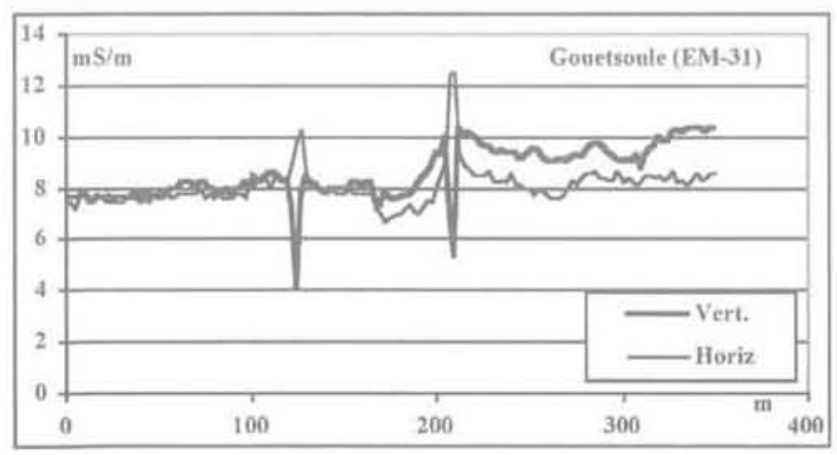

FG. 11 Profils électromagnétiques. Electromagnetic cross section.
4.4

\section{Sondages électromagnétiques en domaine temporel (TDEM)}

Cette méthode, applicable depuis peu aux études de subsurface, présente des avantages à différents points de vue. Comme toutes les méthodes électromagnétiques elle est plus sensible aux formations conductrices qu'aux niveaux résistants, ce qui, ici, peut être intéressant dans la mesure où, nous l'avons vu, la surface de glissement est généralement plus conductrice. Par ailleurs, la profondeur d'investigation est au moins de l'ordre des dimensions de la boucle d'émission, et suivant les résistivités rencontrées jusqu'à trois fois cette dimension (Kamenetsky et al., 2000). Enfin le faible encombrement matériel permet de l'utiliser sur des sites peu accessibles. Par sa topographie escarpée et son manque d'accessibilité, le glissement des Gentianes se prêtait bien à ce type de mesures. Douze sondages y ont été réalisés avec une boucle de $25 \mathrm{~m}$ de côté (Fig. 12).

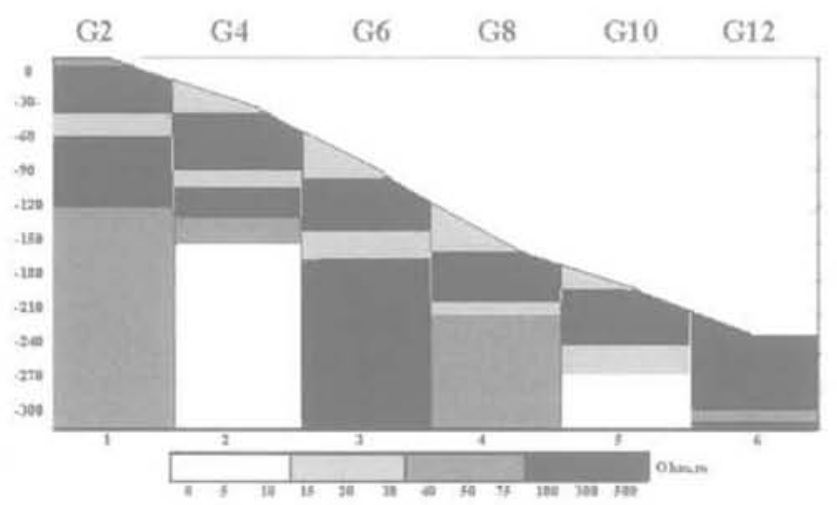

FG. 12 Sondage TDEM sur le site de la Gentiane. Coupe longitudinale dans laxe du glissement de la Gentiane (G2, G4, G6, G8, G10 et G12).

Gentiane TDEM array. Gentiane landsłide longitudinal cross (G2, G4, G6, G8, G10 and G12).

Les résultats sont obtenus par inversion à l'aide du logiciel Tem-Res (Fainberg et Barsukov, 1999), la convergence du processus et la cohérence des résultats nécessitent une bonne approximation en mode interactif.

Dans ces conditions, sur les coupes, les valeurs de résistivité et surtout les épaisseurs des niveaux situés au-dessous de 60-75 $\mathrm{m}$ par rapport à la surface sont à prendre avec précaution. Les douze sondages peuvent être regroupés en quatre familles se différenciant par la succession et l'épaisseur interprétée des couches. En surface nous trouvons un niveau moyennement résistant dont l'épaisseur n'est faible qu'à l'aval. Au-dessous, se présente un horizon résistant à très résistant 500 à $3500 \Omega$ m, avec une forte épaisseur $(\approx 30 \mathrm{~m})$, surmontant dans tous les cas une zone conductrice de 20 à $70 \Omega$.m, dont l'épaisseur de 10 à $25 \mathrm{~m}$ ne peut être définie avec précision.

Enfin, les sondages se terminent tous dans une formation résistante de 300 à plus de $500 \Omega$.m. 
TABLEAU1 Résultats des sondages électromagnétiques. Electromagnetic survey results.

\begin{tabular}{|c|c|c|c|c|c|c|c|}
\hline \multicolumn{2}{|c|}{$1-\mathrm{G} 2, \mathrm{G} 3, \mathrm{G} 7$} & \multicolumn{2}{|c|}{ 2-G4, G5, G6 } & \multicolumn{2}{|c|}{ 3-G9, G10 } & \multicolumn{2}{|c|}{ 4-G11, G12 } \\
\hline Rho $(\Omega, \mathrm{m})$ & $\mathrm{H}(\mathrm{m})$ & Rho $(\Omega . \mathrm{m})$ & $H(m)$ & Rho $(\Omega . \mathrm{m})$ & $\mathrm{H}(\mathrm{m})$ & Rho $(\Omega . \mathrm{m})$ & $\mathrm{H}(\mathrm{m})$ \\
\hline $110-140$ & $7-9,5$ & $150-190$ & $11-14$ & $65-75$ & $1-2,5$ & $500-700$ & $27-35$ \\
\hline $400-600$ & $36-38$ & $3600-5000$ & $27 \cdot 40$ & 2000 & $40-50$ & $2000-2150$ & $35-45$ \\
\hline $60-90$ & $10-20$ & $470-520$ & $4-20$ & $20-60$ & & $30-80$ & \\
\hline 3000 & & $40-50$ & 10 à 20 & 2000 & & 2500 & \\
\hline & & 500 à 2000 & & & & & \\
\hline
\end{tabular}

Nous remarquons également que les niveaux les plus résistants se trouvent dans la partie moyenne du glissement (G4, G5, G6) ce qui correspond aussi à la pente la plus forte.

L'épaisse formation résistante correspondrait à la masse déplacée, tandis que la couche la plus conductrice représenterait la base du glissement, dont la teneur en eau serait plus élevée, au contact du substratum résistant.

\section{5}

\section{Conclusion}

Nous avons, au travers de cette note, présenté les différents facteurs caractéristiques des glissements de terrain observables par mesures indirectes. En cela, l'approche par des méthodes géophysiques a permis d'évaluer l'épaisseur des sédiments engagés dans des glissements, d'en préciser leurs limites géométriques, de mettre en évidence la présence d'un système de che- nalisation interne favorisant l'alimentation en eau interne du glissement mais aussi les surpressions interstitielles déstabilisatrices et de confirmer l'existence d'un cryptorelief. L'utilisation conjointe de différentes méthodes doit être constamment développée, certaines d'entre elles se distinguant par leur rapidité et/ou la légèreté des équipernents (EM, TDEM), ou par l'intérèt de leurs réponses (résistivité). D'autres caractéristiques liées aux glissements de terrain peuvent être atteintes par des méthodes géophysiques telles que la sismique réfraction, sismique réflexion ou radar. La légèreté des matériels, nécessaires à la mise en ceuvre de certaines méthodes, rend celles-ci mieux adaptées aux terrains accidentés. Ces méthodes non destructives ne sont pas suffisantes en soi et elles peuvent être accompagnées par la pose de piézomètres, ce qui permet de préciser les conditions hydrauliques utilisées dans les calculs de stabilité. Enfin, la localisation de drains d'alimentation en eau, non visibles à l'affleurement, mais présents dans le sol, permet d'envisager à plus long terme une utilisation systématique de profils de résistivités pour une meilleure analyse des glissements de terrain.

\section{$\overline{\text { Bibliographie }}$}

Bogaard T.A., Van Asch T.W.J. - "Geophysical and hydrochemical investigation of a complex large-scale landslide in southern France D. 8th International Symposium on Landslides, Norvège, 1996, p. 643-647.

Bogoslovsky V.A., Ogilvy A.A. - $\alpha$ Geophysical methods for the investigation of landislides D. Geophysics vol. $42, \mathrm{n}^{\circ} 3$. 1977. p. $562-571$.

Caris J.P.T., Van Asch T.W.J, - - Geophysical, geotechnical and hydrological investigations of a small landslide in French Alps 1. Engineering Geology $n^{\circ}$ 31. 1991, p. 249.276.

Coussot P. - $\approx$ Rhéologie des boues et laves torrentielles. Etude de dispersions et suspensions concentrées \%. Série montagne $n^{\circ} 5,1993$, CEMAGREF, $413 \mathrm{p}$.

Durville J.-L. - « Mécanisme et modèles de comportement des grands mouvements D. Bull. Inter. Ass. Enqineering Geology, $n^{\circ} 45$, 1992, p. 25-42.

Fabre R., Lebourg T. Clément B. - $"$ Les dépôts morainiques holocènes de la zone axiale pyrénéenne : approche déterministe de leur instabilité dans le secteur de Verdun sur Ariège (Pyrénées centrales) v. Bull. Engineering Geology Environment vol., $n^{\circ} 58,2000$, p. 133-143.
Fainberg E., Barsukov P. - Tem- researcher manual. Netherlands, AEMR, 1999.

Flageolet J.C., Maquaire O., Martin B. Weber D. - "Landslides and climatic conditions in the Barcelonnette and Vars basins (Southern French Alps. Francel n. Geomorphology. Elsevier. n'30, 1999, p. 65-78.

Gallipoli M.R., Lapenne V., Orenzo P. Mucciarelli M. Perrone A.. Piscitelli S., Sdao F. - « Comparison of geological and geophysical prospecting techniques in study of a landslide in southern Itaity n. Eur. $J$. Environmental and Enginneering Geophysics, $n^{\circ} 4,2000$, p. $117-128$.

Kamenetsky F, Oelsner C, $-\alpha$ Distorsion of EM transients in coincidient loops at short time delays n. Geoph. Prosp, vol. 48, $\mathrm{n}^{\circ}$ 6, 2000, p. 983-993.

Lebourg T. - « Analyse qéologique et mécanique de glissements de terrain dans des moraines des Pyrénées centrales et occidentales (France) 1 . Thèse de I'Université Bordeaux 1, 2000, 363 p.

Lebourg T., Fabre R. Clément B. - « Gla. cial tills instability on mountainsides, influence of the geomorphologic inheritance and the heterogeneity, for forecasting the behaviour of slope movements $x .8$ th International Symposium on Landslides, Cardiff, 2000, p. 887-892.
Lebourg T, Frappa M. Sirieix C. a Reconnaissance des surfaces de rup. ture dans les formations superficielles instables par mesures électriques $x$. Pangea, n 31-32, 1999.

Loke M.H. - " Rapid 2D resistivity inversion using the least-squares method $\mathrm{x}$. ABEM Instruments, AB. Sweden, 1996.

McNeil J.D. - a Electromagnetic terrain conductivity measurement at low induction numbers m. Technical note TN-6. Geonics, 1980, 29 p.

Ogilvy A.A. Aved M.A. Bogoslovsky V.A Geophysical studies of water leakages from reservoirs 1. Geophys. Prospec. vol. $17, \mathrm{n}^{\circ} 1,1969, \mathrm{p}, 32-62$.

Ogilvy A.A. Bogoslovsky V.A. - $\alpha$ The possibilities of geophysical methods applied for investigating the impact of man on the geological medium $n$. Geoph. Prosp. vol, $27, n^{\circ} 4,1969$, p. $775-789$.

Sorgi-de-Gennaro C. - "Contribution à l'étude des facteurs climatiques, géolo. giques et géotechniques contrólant la génération de laves torrentielles. Application au secteur de Saint-Martin-de-laPorte (Savoie, France) 》. Thèse ENSMP, 1999. 172 p.

Wong H.N. - a Keynote lecture: Landslides in Hong Kong n. 8th International Symposium on Landslides, Cardiff UK, Telford Ed, 2000. 